\title{
High-resolution charge exchange measurements at ASDEX Upgrade
}

E. Viezzer, ${ }^{1}$, a) T. Pütterich, ${ }^{1}$ R. Dux, ${ }^{1}$ R. M. McDermott, ${ }^{1}$ and the ASDEX Upgrade Team $^{1, b)}$

Max-Planck-Institut für Plasmaphysik, EURATOM Association, Boltzmannstr. 2, 85748 Garching, Germany

( Dated: 15 July 2012)

The charge exchange recombination spectroscopy (CXRS) diagnostics at ASDEX Upgrade (AUG) have been upgraded and extended to provide high-resolution measurements of impurity ion temperature, density and rotation profiles. The existing core toroidal CXRS diagnostic has been refurbished to increase the level of signal, thus enabling shorter exposure times down to $3.5 \mathrm{~ms}$. Additional lines of sight provide more detailed profiles and enable simultaneous measurements of multiple impurities. In addition, a new CXRS system has been installed which allows for the measurement of poloidal impurity ion rotation in the plasma edge with high temporal (1.9 ms) and spatial resolution (down to $5 \mathrm{~mm}$ ). A new wavelength correction method has been implemented to perform in-situ wavelength calibrations on a shot-to-shot basis. Absolute measurements of the poloidal impurity ion rotation with uncertainties smaller than $1.5 \mathrm{~km} / \mathrm{s}$ have been obtained. Comparison of all the CXRS measurements provides a consistency check of the diagnostics and good agreement has been found for all of the CXRS systems.

PACS numbers: 52.55.-s, 52.70.-m, 07.60.Rd, 42.79.e

Keywords: Tokamak, plasma diagnostics, optical systems, spectroscopy, CXRS, ion temperature, rotation

\footnotetext{
a) Electronic mail: Eleonora.Viezzer@ipp.mpg.de

b) For authors' list see A. Kallenbach et al., Nucl. Fusion 51094012 (2011)
} 


\section{INTRODUCTION}

The most common method to measure the rotation in a tokamak plasma is active charge exchange recombination spectroscopy $(\mathrm{CXRS})^{1}$. The light emitted due to charge exchange (CX) reactions between fully ionized impurity ions and injected neutral atoms yields localized information on impurity ion temperature and velocity from the Doppler widths and Doppler shifts of the measured spectra. Impurity densities are obtained from the line intensities of the measured spectra. For the next generation fusion experiment ITER $^{2}$, CXRS will play a crucial role for measuring the density of the fusion ash $\mathrm{He}^{2+}$.

At ASDEX Upgrade $(\mathrm{AUG})^{3}$, toroidal CXRS systems viewing the core and the edge ${ }^{4}$ of the plasma provide temporally and radially resolved CXRS profiles. Recently, the core toroidal CXRS diagnostic was upgraded to obtain more detailed radial profiles and a higher level of signal enabling shorter integration times. In addition, the edge toroidal view of the CXRS systems has been supplemented with a poloidal view. Along with a consistency check of ion temperature and impurity ion density, the diagnostic enables the measurement of the poloidal impurity ion rotation. Thus, it provides the missing measurements for deriving the radial electric field from the radial force balance equation ${ }^{5}$. Furthermore, the combination of toroidal and poloidal views allows the determination of full plasma rotation profiles both parallel and perpendicular to the magnetic field lines.

This paper is organized as follows: In section II the upgrades of the toroidal CXRS diagnostics at AUG are described. The setup of the new poloidal system, along with example profiles in $\mathrm{H}$-mode ${ }^{6}$ plasmas, is presented in section III. The radial plasma sweep technique, which is applied to improve measurements at the plasma edge, is described in section IV. Wavelength corrections as well as corrections due to the CX cross-section and Zeeman effects are discussed in section V. A summary of the paper is given in section VI.

\section{UPGRADE OF TOROIDAL CXRS AT AUG}

At AUG CXRS measurements are routinely made to provide temporally and spatially resolved profiles of impurity ion toroidal rotation, temperature and density. The core CXRS systems on AUG view two different (toroidally separated) heating beams, see figure 1. One of the core systems has recently been upgraded to increase the number of lines of sight (LOS) 

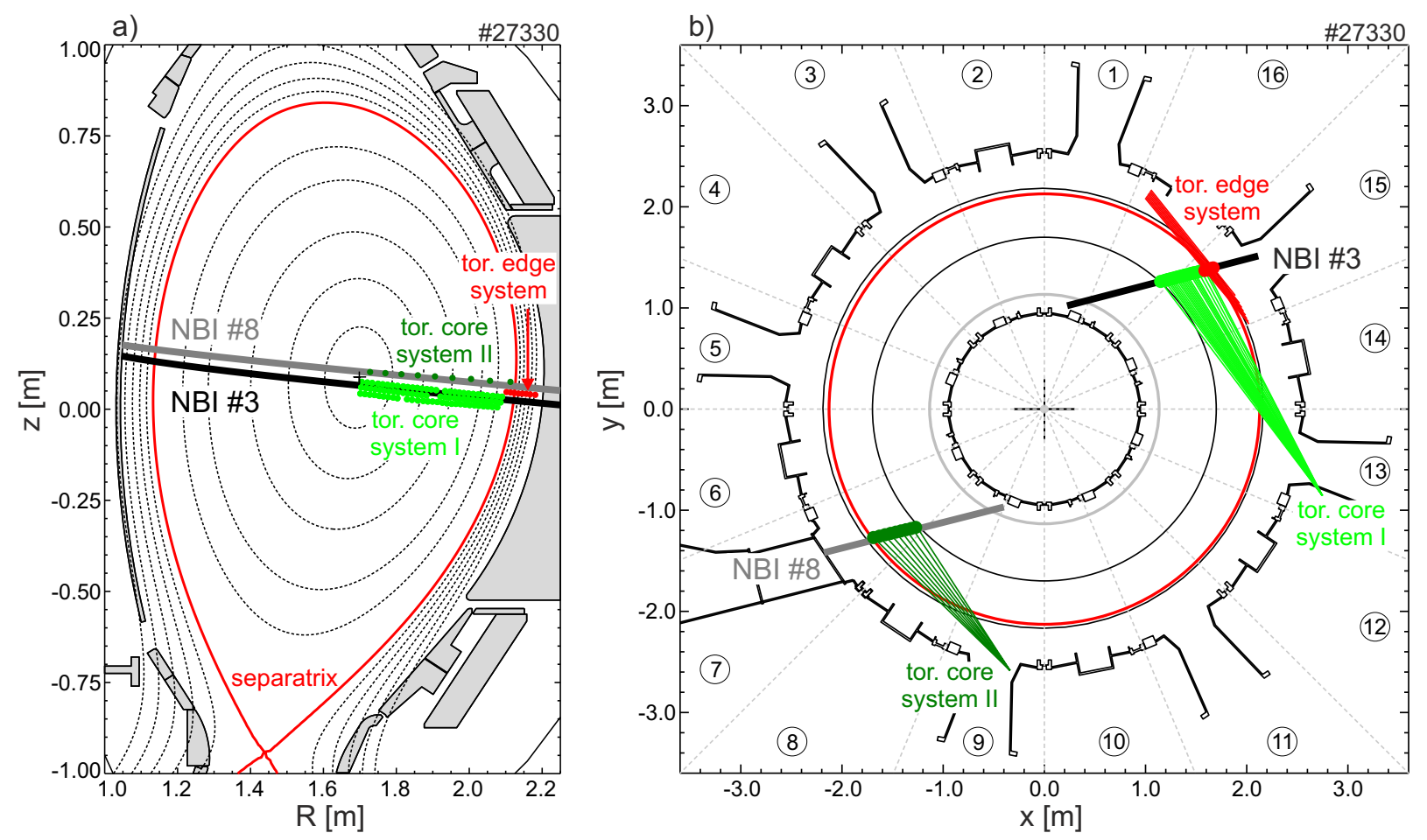

FIG. 1. a) Poloidal cross-section of AUG showing the collection regions of all toroidal CXRS diagnostics, b) top down view of AUG vessel showing the lines of sight of the toroidal CXRS systems. The indicated beam lines do not correspond to the actual width of the beams $(\sim 25 \mathrm{~cm})$.

and the level of signal, thus enabling measurements at shorter integration and repetition times. The diagnostic uses an optical head which is mounted at sector 13 outside of the vessel. The optical setup has been redesigned to install additional LOS, thus allowing more detailed radial CXRS profiles to be measured. The optical head is equipped with 91 high-temperature fibers (diameter of $400 \mu \mathrm{m}$ ) divided into three vertically displaced rows ( 30 fibers/row, see figure 1a)), which are focused on the center of the neutral beam injection (NBI) source \#3 (sector 15) using a Nikon $f / 1.8$ objective lens and an in-vessel $\mathrm{Al}$ mirror (coated with $\mathrm{MgF}_{2}$ ). Where the views intersect the beam the three rows are vertically separated by only $1.6 \mathrm{~cm}$, which is considerably smaller than the size of the heating beam $(\sim 25 \mathrm{~cm})$. Each row of fibers covers a radial range extending from the magnetic axis $(\sim 1.65 \mathrm{~m})$ to the top of the pedestal $(\sim 2.15 \mathrm{~m})$ with a spot size in the focal plane of $1.5 \mathrm{~cm}$ and a radial resolution of $\pm 2-2.5$ $\mathrm{cm}$. The three rows are dedicated to separate diagnostics, with the top row used for CXRS measurements, the central row for beam emission spectroscopy (BES) ${ }^{7}$, and the bottom row for fast-ion $\mathrm{D}_{\alpha}$ (FIDA) measurements ${ }^{8}$. The BES measurements enable the evaluation of the 


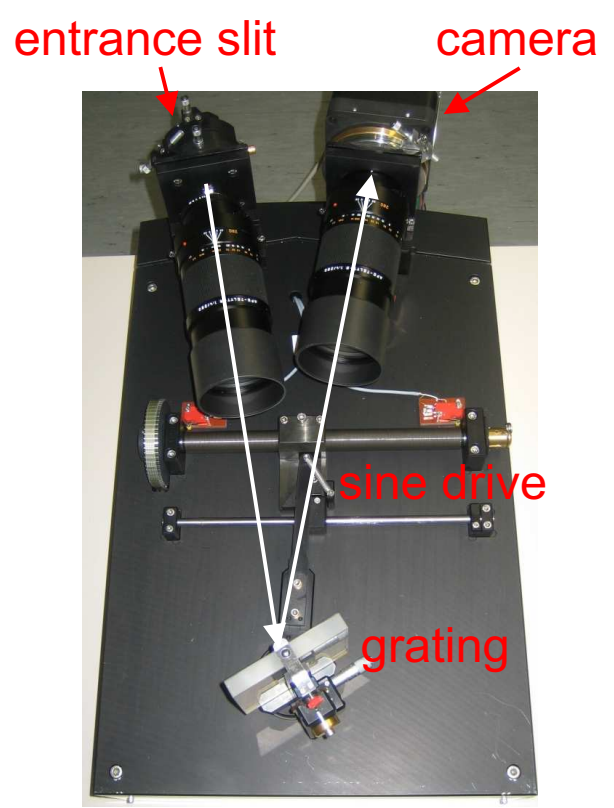

FIG. 2. Setup of a lens-based Czerny-Turner like spectrometer: The fibers, which guide the collected light from the torus to the spectrometer, are stacked vertically along the entrance slit. After passing through the entrance slit the light is collimated, then dispersed by a 2400 grooves/mm grating and then focused onto the camera. The sine drive enables to move the central wavelength of the spectrometer.

beam neutral density along LOS virtually identical to those used for CXRS, thus providing a cross-check on the beam densities evaluated with attenuation codes and improving the accuracy of impurity density profiles calculated from CXRS intensities.

The CX emission collected along the LOS is transmitted to a high throughput $f$ / 4 CzernyTurner like spectrometer, which utilizes a variable width entrance slit, two objective lenses (Leica APO-ELMARIT-R), and a movable grating with 2400 grooves/mm (see figure 2). The spectrometer is coupled to a new Princeton Instruments ProEM, back-illuminated, frametransfer charge coupled device (CCD) camera with on-chip multiplication gain ${ }^{9}$. This allows for low light applications and improves the measurement capability in the plasma core. The camera features a $512 \times 51216 \mu \mathrm{m}$ pixel chip which, when coupled with the spectrometer, enables a $13.1 \mathrm{~nm}$ spectral range at a central wavelength of $494.5 \mathrm{~nm}$. The entrance slit to the spectrometer is typically set to $100 \mu \mathrm{m}$, but can be adjusted to any value between 0 and $400 \mu \mathrm{m}$. For a slit width of $100 \mu \mathrm{m}$ and a central wavelength of $494.5 \mathrm{~nm}$ the instrumental width of the system is of the order 0.067 to $0.074 \mathrm{~nm}$ (slight variations of the different 
spectrometer channels arise due to imperfections of the entrance slit). The shape of the instrument functions for the slit widths used $(\leq 100 \mu \mathrm{m})$ is well described by a Gaussian function. Hence, the deconvolution can be performed analytically in the fitting process and the measured width of the spectrum has contributions from the instrumental width and the Doppler width. The collimating lens at the entrance slit has a focal length of 280 $\mathrm{mm}$, while the focusing lens has a focal length of $180 \mathrm{~mm}$. This choice of lenses introduces a de-magnification factor of 1.56 and enables the light from 25 fibers, which are stacked vertically along the entrance slit, to be imaged concurrently on the camera. Compared to a conventional Czerny-Turner spectrometer the main advantages of using the optical layout of a lens-based Czerny-Turner like spectrometer are the good imaging properties of the system at high aperture (no astigmatism) and at the same time, the simultaneous measurement of many channels.

The ability to move the central wavelength of the spectrometer enables the system to make CX measurements of different impurity species. The system typically utilizes either the $\mathrm{B}^{5+}(n=7 \rightarrow 6) \mathrm{CX}$ line at $494.467 \mathrm{~nm}$ or the $\mathrm{C}^{6+}(n=8 \rightarrow 7)$ line at 529.059 nm. However, it has also been used for He, N, Ne and Ar. Typically, the core toroidal CXRS system runs with an exposure time of $10 \mathrm{~ms}$, but it can be operated down to $3.5 \mathrm{~ms}$ allowing for intrinsic rotation measurements through the use of short beam blips ${ }^{10}$. The new upgrades enable now more detailed studies of toroidal momentum transport ${ }^{11,12}$, impurity transport $^{10}$ and MHD instabilities.

The second core CXRS system is equipped with an in-vessel optical head located in sector 9 (see figure 1b)) which views the NBI source \#8 (sector 7). This system has 10 LOS evenly distributed between the magnetic axis and the top of the pedestal. The LOS of this system are not as tangential to the magnetic flux surfaces as those in sector 13 and thus have poorer radial resolution $( \pm 5-6 \mathrm{~cm})$. Fibers with a diameter of $400 \mu \mathrm{m}$ guide the collected light from this system to a conventional $f / 6.5$ Czerny-Turner spectrometer which employs a movable grating with 2400 grooves $/ \mathrm{mm}$ and two mirrors with a focal length of $500 \mathrm{~mm}$. The entrance slit is adjustable, but is typically set to $100 \mu \mathrm{m}$ and therefore the instrumental widths of the channels are ranging between 0.038 and $0.058 \mathrm{~nm}$ (at a central wavelength of $494.5 \mathrm{~nm}$ ). The measured spectra are analyzed by a Princeton Instruments MicroMax camera, which has a back-illuminated CCD chip with $512 \times 51213 \mu$ m pixels allowing for a spectral range of $3.5 \mathrm{~nm}$ at a central wavelength of $494.5 \mathrm{~nm}$. The CCD camera is operated 

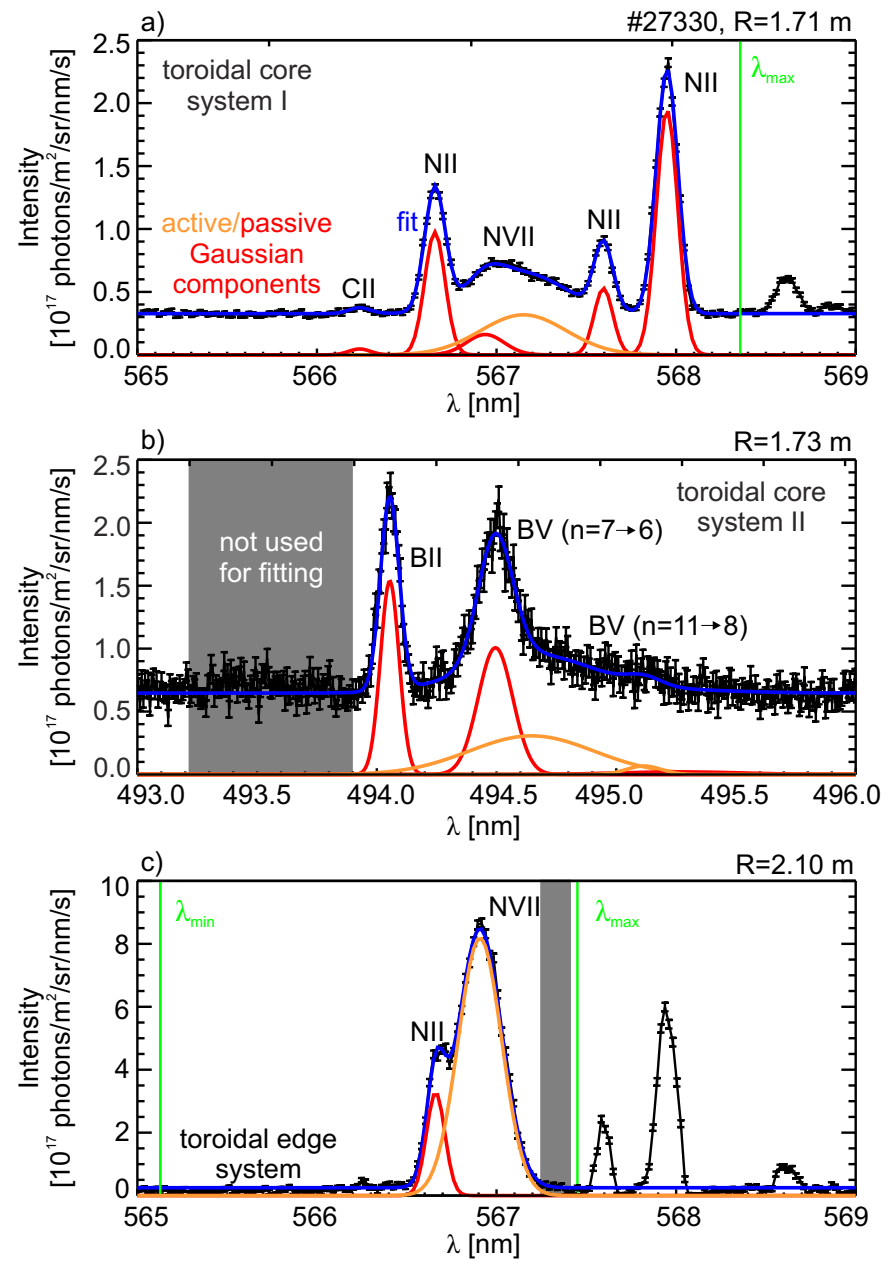

FIG. 3. Example spectra of toroidal CXRS diagnostics: a) $\mathrm{N}^{7+}$ spectrum of core system I, b) $\mathrm{B}^{5+}$ spectrum of core system II, c) $\mathrm{N}^{7+}$ spectrum of edge system. The areas highlighted in gray as well as those larger (smaller) than $\lambda_{\max }\left(\lambda_{\min }\right)$ are not used for fitting the spectra.

in frame-transfer mode. Binning the CCD chip to 9 regions of interest allows for a temporal resolution of $7.5 \mathrm{~ms}$. However, the diagnostic is typically set to $20 \mathrm{~ms}$ due to the signal to noise ratio. The poor signal to noise ratio arises from the degraded in-vessel optics. For experiments in which the NBI source \#8 is not utilized the spectrometer is equipped with extra LOS from the optical head of the toroidal core CXRS system I. This enables the measurement of two different impurity species on the same beam and a cross-check on intensity and wavelength calibrations when imaging the same impurity. Figure 3a) and b) show typical spectra obtained with the core toroidal CXRS systems. The fit to the spectrum is shown in blue, while the Gaussian components are plotted in orange (active lines) and red (passive lines). In this discharge, the toroidal core system I measured the $\mathrm{N}^{7+}(n=9 \rightarrow 8$, 
$\lambda=566.937 \mathrm{~nm}$ ) spectral line. The spectra are fitted with 6 Gaussians; one Gaussian for the active $\mathrm{N}^{7+}$ line, one for its passive component, 3 Gaussians accounting for 3 nearby passive $\mathrm{N}^{1+}$ lines at 566.663, 567.602 and $567.956 \mathrm{~nm}$ and one Gaussian for a passive $\mathrm{C}^{1+}$ line at $566.246 \mathrm{~nm}^{13}$. A discrimination between electron impact excitation and charge exchange with thermal neutral deuterium ${ }^{14}$ is not applied for the passive component as it is typically small compared to the active CX line. In this discharge the spectrometer of the core CXRS system II utilized LOS from the core CXRS system I (i.e. viewing NBI source \#3 (see figure 1)) and measured the $\mathrm{B}^{5+}$ spectral line. The measured spectra are fitted with 5 Gaussians; one accounting for the active $\mathrm{B}^{5+}(n=7 \rightarrow 6)$ line at $494.467 \mathrm{~nm}$, one for the active $\mathrm{B}^{5+}$ component $(n=11 \rightarrow 8)$ at $495.087 \mathrm{~nm}$, two for their passive components and one Gaussian to fit the passive $\mathrm{B}^{1+}$ line at $494.038 \mathrm{~nm}$.

Independently, the toroidal edge CXRS diagnostic has also been upgraded to increase the intensity level. The system has been equipped with a new spectrometer and a new CCD camera (identical to those used by the core system I) enabling 25 LOS to be imaged on the chip simultaneously with a repetition time of $2.65 \mathrm{~ms}$. Shorter exposure times are obtained by using less LOS and binning the CCD chip to fewer regions of interest. For the edge diagnostic, the entrance slit to the spectrometer is typically set to $50 \mu \mathrm{m}$. For this slit width the instrumental width of the spectrometer channels is in the range of 0.031 to 0.036 $\mathrm{nm}$ (at a central wavelength of $494.5 \mathrm{~nm}$ ). The toroidal edge system uses an in-vessel $f / 4$ optical head which images the plasma edge at the outer midplane onto 8 LOS. The LOS are separated by about $1 \mathrm{~cm}$ and each LOS is equipped with 3 optical fibers (with a diameter of $400 \mu \mathrm{m})$ to improve the signal. At the outer midplane $(R=2.15 \mathrm{~m})$ the toroidal curvature of the plasma across the width of the heating beam $(\sim 25 \mathrm{~cm})$ is significant. For this reason a separate $\mathrm{Al}$ mirror coated with $\mathrm{MgF}_{2}$ was used for each LOS (see figure 4) to optimize the radial resolution down to $3 \mathrm{~mm}$, which is obtained when a radial sweep of the plasma (see section IV) is performed. Without this sweep, the radial resolution is given by the channel spacing, i.e. $\sim 1 \mathrm{~cm}$. In the plasma edge of $\mathrm{AUG}(T=500 \mathrm{eV}, B=2 \mathrm{~T})$, the typical Larmor radii of impurity species are of the order $\sim 1 \mathrm{~mm}$ and thus, smaller than the radial resolution.

An example spectrum of the toroidal edge CXRS diagnostic is shown in figure 3c). Here, the edge system was set to the $\mathrm{N}^{7+}$ spectral line. The $\mathrm{N}^{7+}$ spectra are typically fitted with 2 Gaussians, one accounting for the active $\mathrm{N}^{7+}$ line and one for a nearby $\mathrm{N}^{1+}$ line. For the 


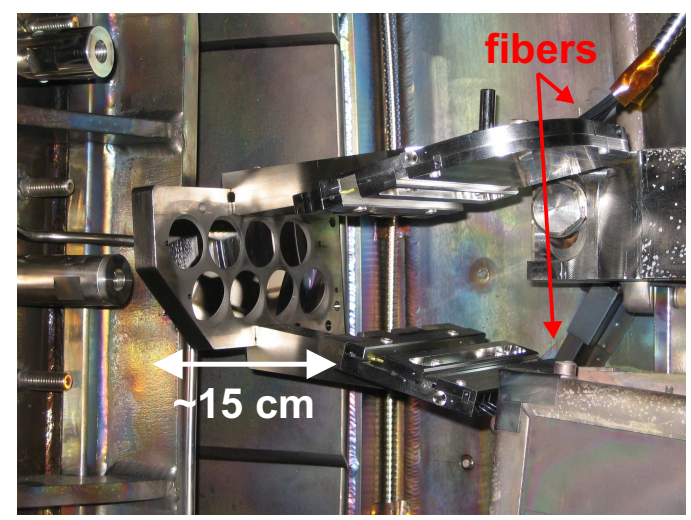

FIG. 4. In-vessel view of the optical head dedicated to toroidal edge CXRS measurements: For each LOS a separate mirror is used to optimize the radial resolution of the system down to $3 \mathrm{~mm}$.

edge system the passive $\mathrm{N}^{6+}$ component is negligible as it is very small compared to the active CX line. Note the difference in scale when comparing the intensities between the core and edge systems, which reflects mainly the attenuation of the beam density. At the plasma edge the intensity of the active line is $2.5 \cdot 10^{17}$ photons $/ \mathrm{m}^{2} / \mathrm{sr} / \mathrm{s}$ while the full width at half maximum (FWHM) is $0.25 \mathrm{~nm}$ (corresponding to an ion temperature of $T_{i}=0.56 \mathrm{keV}$ ) and the absolute wavelength shift of the spectral line is $0.03 \mathrm{~nm}$ (equal to a rotation of $v_{\text {rot }}=15.6$ $\mathrm{km} / \mathrm{s}$ ). In the core the signal is much lower due to the attenuation of the neutral beam. For the spectrum shown in figure 3a) the intensity of the active line is $1.9 \cdot 10^{16}$ photons $/ \mathrm{m}^{2} / \mathrm{sr} / \mathrm{s}$, the FWHM of the spectral line is $0.54 \mathrm{~nm}\left(T_{i}=2.17 \mathrm{keV}\right)$ while the wavelength shift is 0.19 $\mathrm{nm}\left(v_{\text {rot }}=103.9 \mathrm{~km} / \mathrm{s}\right)$.

The toroidal edge CXRS diagnostic typically images 20 channels enabling a time resolution of $2.2 \mathrm{~ms}$. One channel is always dedicated to a wavelength calibration measurement. This channel is connected to a neon lamp which provides a wavelength calibration on a shot-to-shot basis (see section V A). It should be noted that the requirements for temporal and spatial resolution at the plasma edge are considerably more demanding due to the fast transport phenomena and steep gradients. This is one basic reason why special attention is necessary for the plasma edge measurements. The edge profiles may be combined with those measured in the core to obtain full radial profiles, i.e. from the magnetic axis out to the separatrix. Figure 5 shows example profiles of toroidal rotation, $v_{t o r}$, and ion temperature, $T_{i}$, for one time point. The profiles are plotted against the normalized poloidal flux coordinate $\rho_{\text {pol }}$. The data points resulting from the fits in figure 3 are highlighted in gray. In 


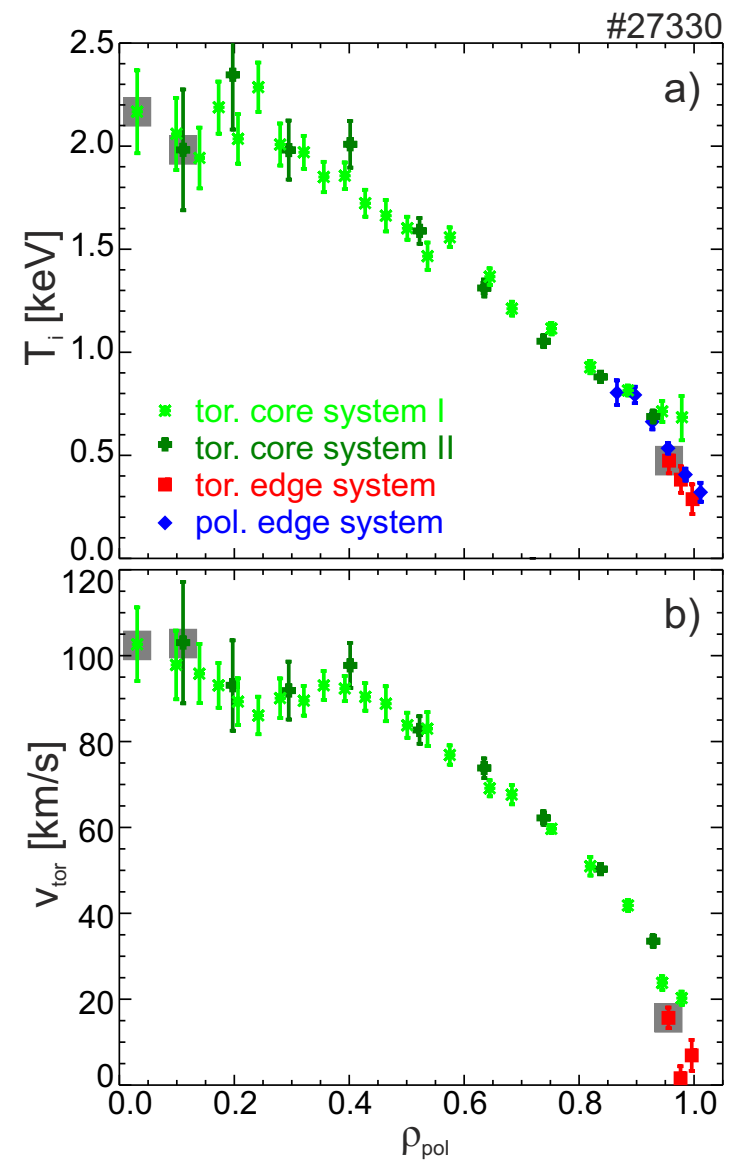

FIG. 5. a) Ion temperature, $T_{i}$, profile obtained from toroidal systems and new poloidal CXRS diagnostic (see section III), b) toroidal rotation, $v_{t o r}$, profile. The data points marked in gray result from the fits to the measured spectra shown in figure 3 .

addition, the ion temperature profile measured by the new poloidal CXRS diagnostic (see section III) is shown in the upper plot of figure 5. Good agreement in $T_{i}$ and $v_{\text {tor }}$ is obtained for all CXRS systems.

\section{POLOIDAL EDGE CXRS DIAGNOSTIC AT AUG}

A newly installed edge poloidal CXRS system also utilizing beam 3 in sector 15 enables the measurement of poloidal impurity ion rotation profiles. The diagnostic has been designed to obtain high spatial resolution (down to $5 \mathrm{~mm}$ ) measurements of the plasma edge at AUG. The system features an $f / 2.5$ optics mounted inside the vessel and employs one lense with a focal length of $40 \mathrm{~mm}$. Note that the radial resolution had to be compromised (by $2 \mathrm{~mm}$ compared 

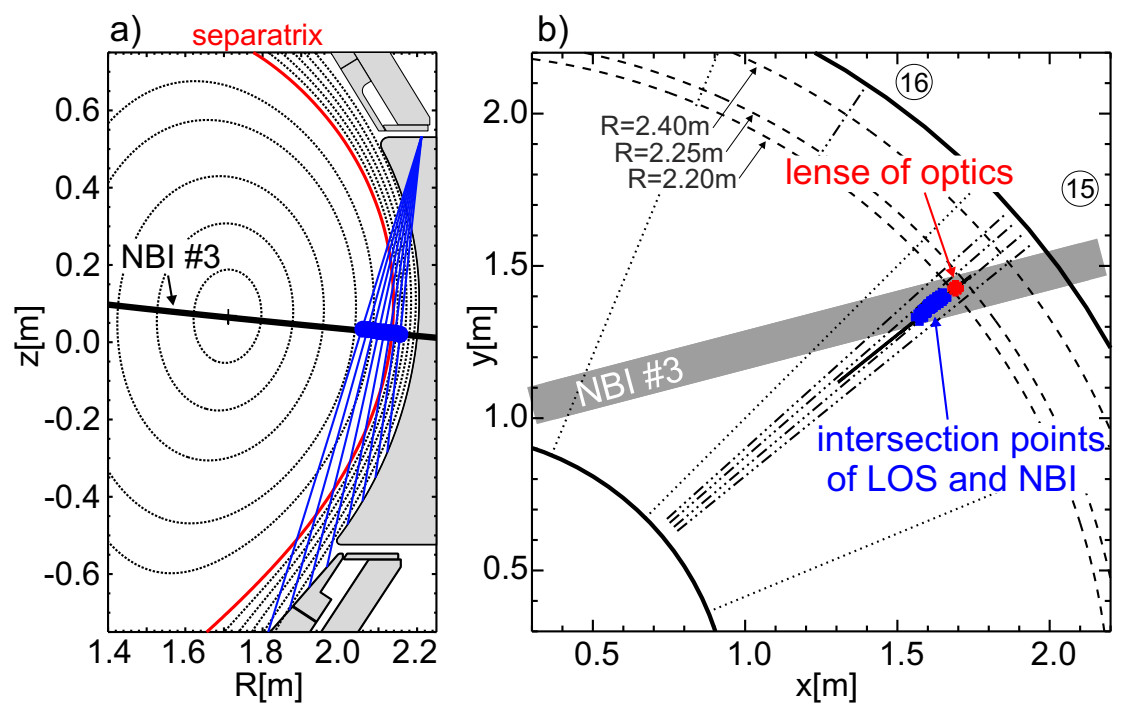

FIG. 6. a) Poloidal cross-section of AUG indicating the location of the poloidal LOS, b) top down view of sector 15 . Note that the beam line does not correspond to the actual size of the beam.

to the toroidal edge system) due to spatial restrictions inside the vessel. The optical head has 8 LOS which view the plasma edge. Each LOS is comprised of two fibers to enhance the signal. In the focal plane the radial distance between two adjacent LOS is $1.25 \mathrm{~cm}$ while the spot size is $5 \mathrm{~mm}$. The in-vessel optical head has been aligned such that the $8 \mathrm{LOS}$ view the edge pedestal in a poloidal plane and to ensure that no toroidal rotation component is picked up. Figure 6 shows an overview of the viewing geometry of the new system. In figure 6a) a poloidal cross-section of AUG is shown along with the LOS of the poloidal diagnostic and the path of the neutral beam. Figure $6 \mathrm{~b}$ ) shows the focal points of the poloidal system with respect to the path of the neutral beam in a toroidal cross-section. The collected light is transmitted to a high-throughput $f / 4$ Czerny-Turner like spectrometer, which is similar to the spectrometer used for the edge toroidal system. It employs two objective lenses with a focal length of $280 \mathrm{~mm}$. Thus, the light from 15 fibers can be imaged simultaneously. The entrance slit of the spectrometer is typically set to a width of $50 \mu \mathrm{m}$. At this slit width and at a central wavelength of $494.5 \mathrm{~nm}$ the instrumental width of the system is between 0.023 to $0.027 \mathrm{~nm}$ depending on the spectrometer channel. The collected photons are imaged onto a frame-transfer back-illuminated CCD camera (Princeton Instruments PhotonMax, $512 \times 512$ $16 \mu \mathrm{m}$ pixels) capable of an electron multiplication readout. The CCD chip, coupled with the spectrometer, allows for a spectral range of $8.2 \mathrm{~nm}$ at a central wavelength of $494.5 \mathrm{~nm}$. 


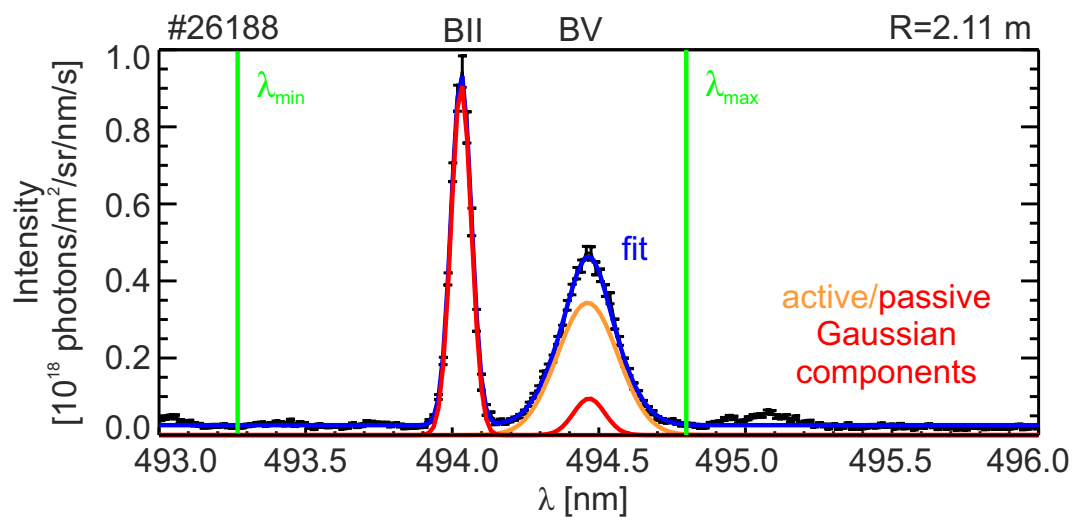

FIG. 7. Example spectrum of the $\mathrm{B}^{5+} \mathrm{CX}$ line measured with the poloidal edge CXRS system. The spectrum is fitted with three Gaussians (one accounting for the active $\mathrm{B}^{5+}$ line, one for the passive component and one for the passive $\mathrm{B}^{1+}$ line); fit in blue, Gaussian components in orange (active line) and red (passive lines); the green lines mark the wavelength range used for the fit.

The spectra from 12 out of 16 fibers, which are connected to the optical head, are routinely measured with a repetition time down to $1.9 \mathrm{~ms}$, while 2 channels of the spectrometer are connected to a neon lamp to provide a wavelength calibration measurement (see section $\mathrm{VA}$ ). The poloidal system is typically set to $2.2 \mathrm{~ms}$ to obtain concurrent measurements with the toroidal edge CXRS diagnostic. Figure 7 shows an example spectrum of the $\mathrm{B}^{5+}$ spectral line along with the fit which includes a background and a passive CX component $\left(\mathrm{B}^{4+}, n=7 \rightarrow 6\right)$. The spectrum is fitted with 3 Gaussians; one accounting for the active $\mathrm{B}^{5+}$ line, one for the passive component and one Gaussian for a nearby passive $\mathrm{B}^{1+}$ line at a rest wavelength of $494.038 \mathrm{~nm}$. The lines marked in green in figure 7 show the wavelength range used in the fitting procedure.

Figure 8 shows poloidal rotation, temperature and density profiles of the $\mathrm{B}^{5+}$ spectral line measured during the inter-ELM (Edge Localized Mode ${ }^{15}$ ) phase of an H-mode plasma. In this discharge a magnetic field on-axis of $-2.5 \mathrm{~T}$, plasma current of $1 \mathrm{MA}$, NBI heating of $5 \mathrm{MW}$ and electron cyclotron resonance heating of $0.8 \mathrm{MW}$ were applied. Note that the sign convention in this paper is as follows: The toroidal magnetic field $\mathrm{B}_{\phi}$ is negative (i.e. clockwise viewed from above), the poloidal magnetic field $\mathrm{B}_{\theta}$ is positive (i.e. pointing downward at the outboard midplane), the plasma current and NBI are in the positive toroidal direction (i.e. counter-clockwise viewed from above). Using this convention, the ion diamagnetic drift direction is positive. 
High-resolution CXRS at ASDEX Upgrade
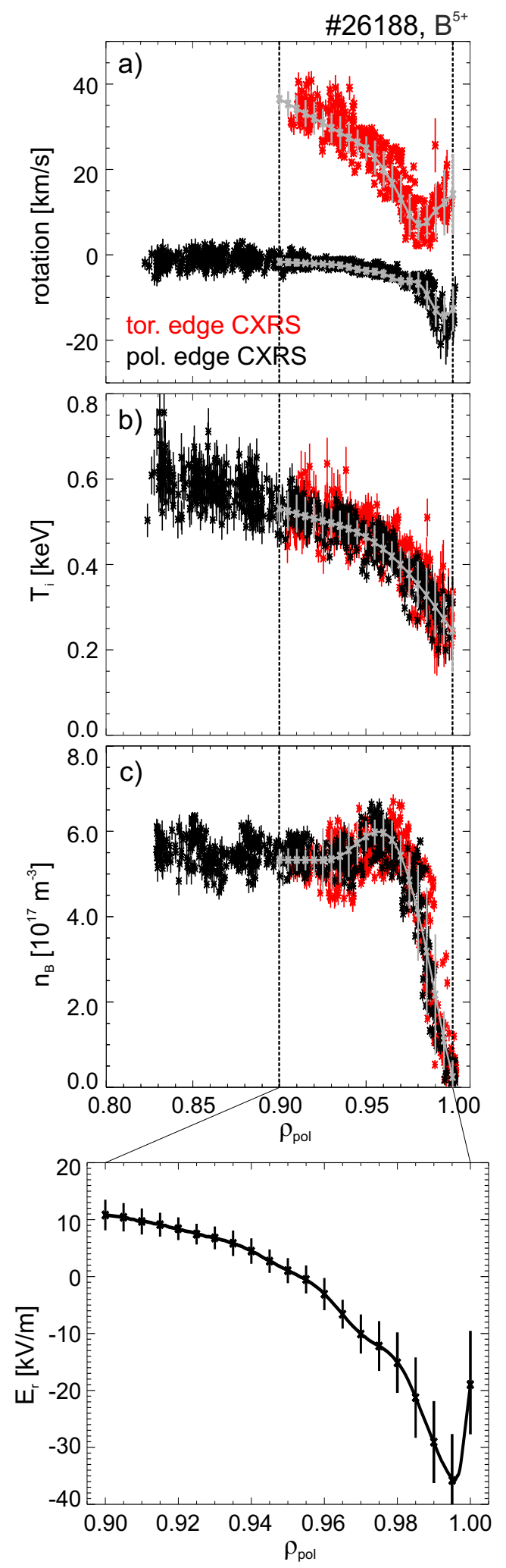

FIG. 8. CX measurements in H-mode: a) poloidal and toroidal rotation, b) ion temperature, c) $\mathrm{B}^{5+}$ density; the measurements obtained from the poloidal diagnostic are illustrated in black, while data from the toroidal system are shown in red. In d) the resulting $\mathrm{E}_{r}$ profile is shown. 
The profiles illustrated in figure 8 are ELM-synchronized, i.e. data acquired during the occurrence of an ELM has been excluded. ELMs cause transient changes to the emission of the spectral line and to the resulting CX profiles and thus, should be removed. Data obtained with the poloidal optical head are shown in black, while the measurements in red result from the toroidal edge system. Furthermore, the radial plasma sweep technique (see section IV) was applied to obtain full radial profiles of the edge transport barrier (ETB $)^{6}$ region. Note that the profiles shown in figure 8 correspond to averaged profiles using data from the whole plasma scan, while the fits to the data are shown in gray. Impurity ion density and temperature profiles show a clear H-mode pedestal. The poloidal impurity ion rotation velocity is very low towards the plasma core, while inside the ETB a strongly sheared rotation in the electron diamagnetic direction is observed consistent with measurements on other devices, such as DIII-D ${ }^{16-18}$, Alcator C-Mod ${ }^{19}$, JET ${ }^{20-22}$, TFTR ${ }^{23}$, TEXTOR ${ }^{24}$, $\mathrm{MAST}^{25}, \mathrm{NSTX}^{26}$ and $\mathrm{LHD}^{27}$.

Combining the poloidal and toroidal measurements enables the determination of the radial electric field, $\mathrm{E}_{r}$, using the radial force balance equation ${ }^{5}$. In figure $8 \mathrm{~d}$ ) the resulting $\mathrm{E}_{r}$ profile is shown. Note that the CX profiles have been aligned relative to the separatrix position and to the electron temperature, $\mathrm{T}_{e}$, and density, $\mathrm{n}_{e}$, measurements (see section IV).

\section{RADIAL PLASMA SWEEP TECHNIQUE TO IMPROVE EDGE CXRS MEASUREMENTS}

To obtain the best radial resolution of the edge CXRS measurements and hence, detailed edge profiles a radial sweep of the plasma edge through the LOS of the diagnostic is performed. Typically, the plasma position is moved by $2 \mathrm{~cm}$ in about $800 \mathrm{~ms}$ during a steady-state portion of the discharge. The radial scan also allows the refinement of the relative sensitivity calibration of different LOS of the edge CXRS diagnostics as slight differences in the calibration show up as discontinuities in the intensity profiles, after mapping the measurements onto the plasma coordinate system $\rho_{\text {pol }}$. Furthermore, this technique enables the alignment of the toroidal and poloidal edge systems via the ion temperature and intensity profiles. In figure 9a-d) several plasma parameters from an $\mathrm{H}_{\text {-mode }}{ }^{6}$ discharge, which includes a radial plasma sweep, are shown. The time period highlighted in yellow 

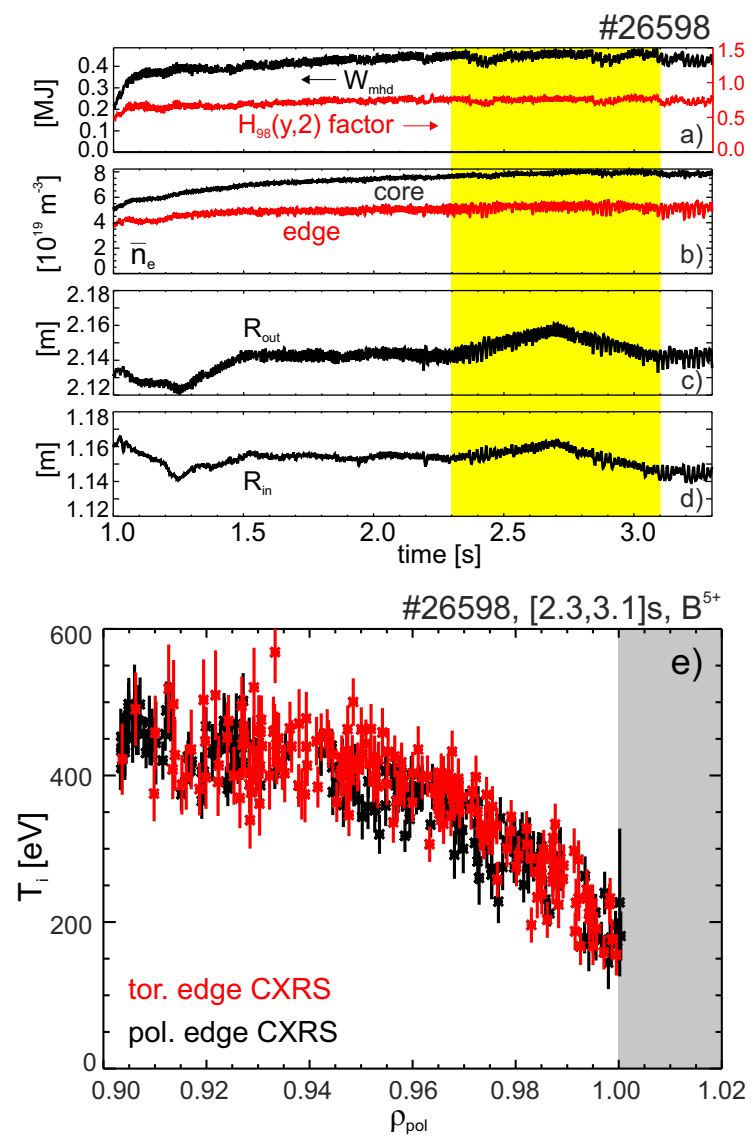

FIG. 9. Plasma parameters from an H-mode discharge that includes a radial plasma sweep: a) plasma stored energy $\mathrm{W}_{m h d}$ (black) and $\mathrm{H} / \mathrm{L}$ scaling factor $\mathrm{H}_{98}(\mathrm{y}, 2)$ with respect to the ITER confinement scaling ${ }^{34}$ (red), b) line averaged electron density in the plasma core (black) and edge $($ red $)$, c) radial plasma position at outer midplane, d) radial plasma position at inner midplane, e) ion temperature profile plotted against the normalized poloidal flux coordinate $\rho_{\text {pol }}$ obtained during the radial plasma scan highlighted in yellow.

contains the radial scan of the plasma. This technique enables complete, very detailed edge CXRS profiles, such as the ion temperature (see figure 9e)). Note that the $T_{i}$ profile is ELMsynchronized (i.e. data acquired during an ELM have been excluded). All the data measured during a radial plasma sweep are combined to one averaged profile. Care has been taken to reduce uncertainties due to the magnetic equilibrium and to optimize the radial profile alignment ${ }^{28}$ (see e.g. figure 2 of ref. $^{4}$ ). The $T_{e}$ profile measured by Thomson scattering ${ }^{29}$ and the electron cyclotron emission (ECE) diagnostic ${ }^{30}$ is shifted such that $T_{e} \approx 100 \mathrm{eV}$ at the separatrix. This condition arises from power balance and parallel heat transport stud- 
ies using a 1-D heat conduction model ${ }^{31}$. The $n_{e}$ measurements obtained with the lithium beam diagnostic ${ }^{32,33}$ are aligned to the profiles measured by Thomson scattering, which has identical measurement volumes for both $n_{e}$ and $T_{e}$. The $T_{i}$ profile is then aligned relative to the $T_{e}$ profile such that the steep gradient regions overlap each other. The accuracy of the relative profile alignment is $2-3 \mathrm{~mm}$, while the shifts applied for the profiles are within the radial resolution of each diagnostic.

In the scrape-off-layer (SOL, highlighted in gray in figure 9e)) the fit results have been omitted. In this region the fractional abundance of fully stripped impurity ions is very small, while additional background emissions disturb the fitting procedure. Therefore, the fitted CX line does not provide information on the actual impurity properties.

\section{CORRECTIONS TO EDGE CXRS MEASUREMENTS}

At the plasma edge both the toroidal and poloidal rotation velocities are quite low and therefore, very accurate knowledge of the wavelength calibration is needed. For this purpose several wavelength corrections have been documented for the edge CXRS diagnostics and are presented in the next subsection. Furthermore, there are atomic physics effects to the spectra, which can give spurious temperature and rotation measurements if they are not properly taken into account. These include the CX cross-section effect, which will be discussed in subsection VB, and the gyro-motion effect, which is described in subsection V C. Corrections due to the Zeeman effect are discussed in subsection VD.

\section{A. Wavelength corrections}

The small rotation velocity of the impurity ions at the plasma edge requires a very accurate wavelength correction, since systematical uncertainties in the rotation would arise from any error in the wavelength calibration. The optical fibers are stacked vertically in front of the entrance slit of the spectrometer. However, the images of these fibers are not vertically aligned on the CCD chip, rather they appear in a curve, which is well described by a parabola. This curvature arises due to the vertical angle of off-axis point sources to the optical axis and is described in detail in ref. ${ }^{35}$. However, small displacements of the input fibers from a perfectly vertical arrangement lead to small deviations from the 


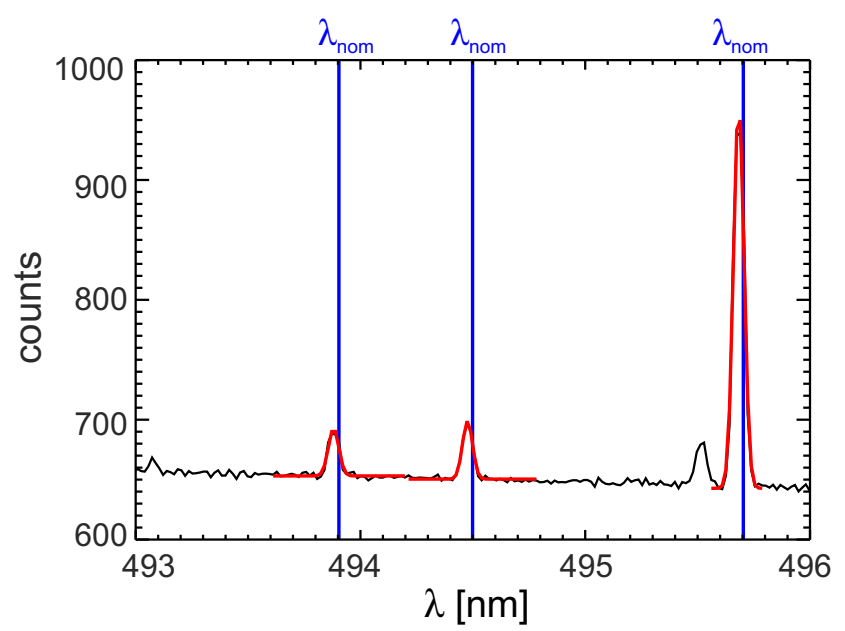

FIG. 10. Example neon spectrum acquired during a discharge, the poloidal edge CXRS diagnostic was set to the $\mathrm{B}^{5+}$ wavelength.

parabola formula. The deviation from the parabolic image of a straight entrance slit of each spectrometer channel has been determined at several different wavelengths using $\mathrm{Hg}$ and $\mathrm{Ne}$ calibration lamps and is taken into account in the analysis of the CX measurements.

For the $\mathrm{B}^{5+}$ line, the wavelength shift associated with a rotation velocity of $1 \mathrm{~km} / \mathrm{s}$ is 1.65 pm. On this scale a systematic change in the Doppler shift may even arise from changes in the air pressure and temperature ${ }^{26}$. Therefore, an additional wavelength calibration is performed on a shot-to-shot basis. Two channels of the spectrometer dedicated to poloidal CX measurements (one channel for the spectrometer of the toroidal edge system) are switched to a neon lamp which allows a neon spectrum to be measured in every discharge. From these spectra, the apparent systematic shift is calculated and the 'true' poloidal rotation velocity is evaluated. This method allows the determination of the wavelength calibration quite accurately with uncertainties smaller than $1 \mathrm{~km} / \mathrm{s}$. Figure 10 shows an example spectrum of the neon lamp acquired during a discharge. The sum of 200 spectra collected at the end of the discharge is used for the calibration. In the vicinity of the $\mathrm{B}^{5+}$ wavelength three well-known neon lines are fitted. The lines, marked in blue in figure 10, represent the nominal value of the neon lines at 493.904, 494.499 and $495.703 \mathrm{~nm}$. The spectral lines are each fitted using one Gaussian. The average of the difference between the central wavelength and the theoretical wavelength of the three neon lines is then used to calculate the apparent systematic shift. In this discharge, the shift of the neon lines is $0.023,0.022$ and $0.021 \mathrm{~nm}$ corresponding to velocities of $13.9,13.3$ and $12.7 \mathrm{~km} / \mathrm{s}$, respectively. Note that this shift is 
mainly given by the mechanical uncertainties of the sine drive, which controls the position of the grating.

A similar wavelength calibration method is used for the core CXRS systems. However, these are not performed on a shot-to-shot basis and are typically only accurate to $\pm 2-3 \mathrm{~km} / \mathrm{s}$.

\section{B. Charge exchange cross-section effect}

Due to the energy-dependent CX cross-sections several atomic physics effects arise when measuring CX emission. The main effect is that an apparent wavelength shift, which is not associated with the Doppler motion of the parent ion distribution, can be observed in the measured spectra ${ }^{36}$. Ions moving towards/away from the beam sample different regions
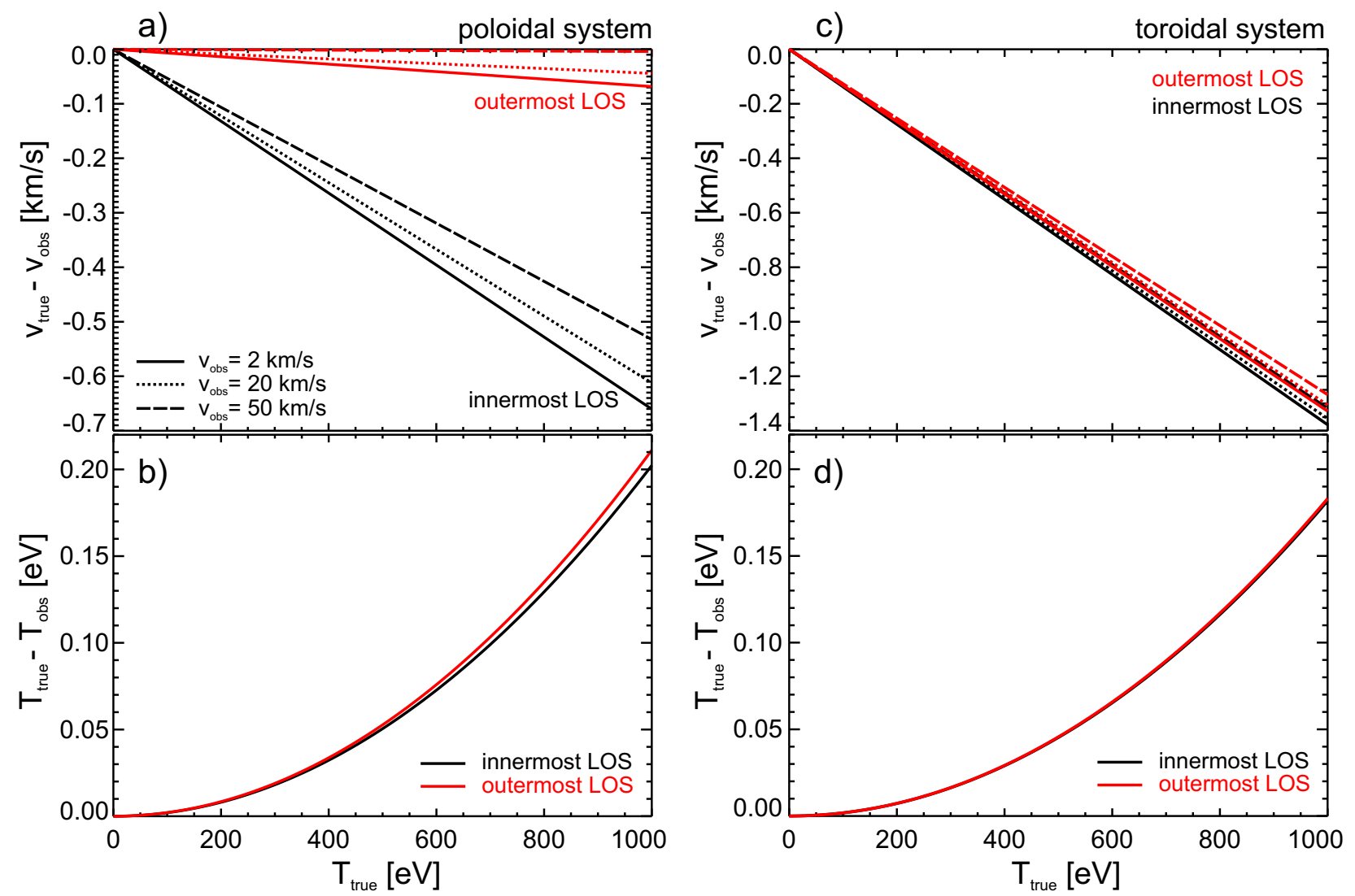

FIG. 11. Cross-section effects on measured $\mathrm{B}^{5+} \mathrm{CX}$ spectra for a $60 \mathrm{keV}$ deuterium beam: a) difference of true and observed velocities, $v_{t r u e}-v_{o b s}$, for poloidal system, b) corrections to observed temperatures, $T_{o b s}$, with respect to the true temperature, $T_{\text {true }}$, for the poloidal diagnostics, c) $v_{\text {true }}-v_{\text {obs }}$ for toroidal optical head, d) $T_{\text {true }}-T_{o b s}$ for toroidal system. 
of the energy-dependent CX cross-sections and thus, have a higher/lower probability to undergo CX. This leads to an enhancement of the observed line intensity on one side of the spectrum, while the other side is reduced. Hence, the spectra are distorted and result in an apparent line broadening and shift not associated with the true ion temperature and velocity. The apparent line shift is due to the first derivative of the cross-section while the apparent line broadening arises due to its second derivative. The cross-section effects on the measured CX spectra have been simulated for the geometry of the edge systems and for a 60 $\mathrm{keV}$ deuterium beam (first, second and third beam energy components) using the approach of von Hellermann et $\mathrm{al}^{36}$. The LOS have a nearly perpendicular view and are aligned in a poloidal (see figure 6) and toroidal (see figure 1) plane, respectively. Figure 11a) and b) show the corrections to the observed temperatures, $T_{\text {obs }}$, and velocities, $v_{o b s}$, with respect to the true temperature, $T_{\text {true }}$, and velocity, $v_{\text {true }}$, for $\mathrm{B}^{5+}$ for the inner- and the outermost LOS

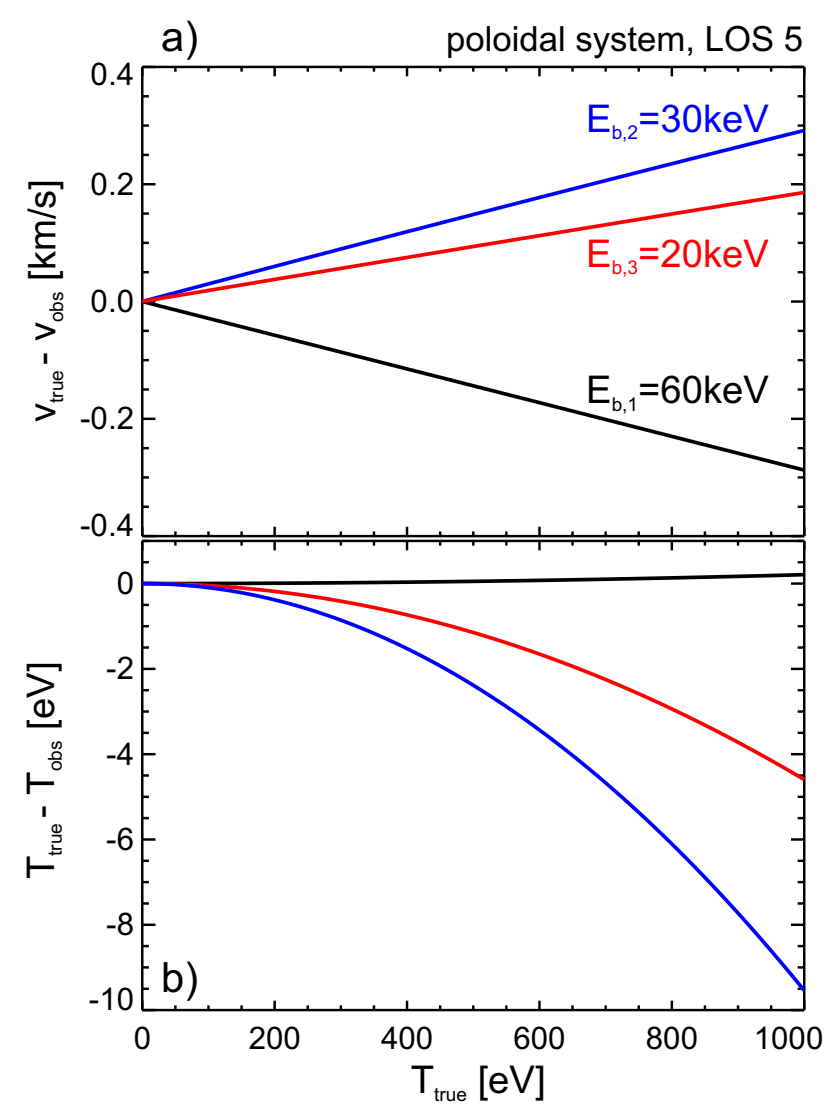

FIG. 12. Cross-section effects in $\mathrm{B}^{5+} \mathrm{CX}$ spectra for first, second and third energy component of a $60 \mathrm{keV}$ deuterium beam: a) difference of true and observed velocities, b) corrections to observed temperatures. 
High-resolution CXRS at ASDEX Upgrade

of the poloidal optical head, while figure 11c) and d) show the corrections for the toroidal edge system. The corrections are well within the error bars of the measurements. Even for an edge rotation of $50 \mathrm{~km} / \mathrm{s}$ the correction due to the energy-dependent CX cross-section is less than $1.5 \mathrm{~km} / \mathrm{s}$ for the toroidal system and less than $0.7 \mathrm{~km} / \mathrm{s}$ for the poloidal system. In the considered temperature range at the plasma edge $(200-500 \mathrm{eV})$ the temperature corrections are between 0.01 to $0.05 \mathrm{eV}$.

Figure 12 shows the corrections for the first, second and third beam energy components of a $60 \mathrm{keV}$ deuterium beam. Both the velocity and temperature corrections of the second and third beam energy components change sign, while the temperature corrections are an order of magnitude higher compared to the correction of the first beam energy component. Note, however, that the first beam energy component contributes about $90 \%$ to the total CX emission, while the contributions of the second and third component are of the order $8 \%$ and $2 \%$, respectively (taking into account the effective CX emission rate and the beam energy population densities, $\left.\mathrm{E}_{0}: \mathrm{E}_{0} / 2: \mathrm{E}_{0} / 3=65 \%: 25 \%: 10 \%\right)$.

The results obtained from these calculations indicate that for the observed temperature and velocity ranges the cross-section effects on the measured spectra are negligible and hence, they are not included in the analysis.

\section{Gyro-motion effect}

In addition to the CX cross-section effect described above, the effect of the gyro-motion of the impurity ion along with the finite lifetime of the observed state ${ }^{37}$ can become important when measuring in the plane of the gyro-orbit. An apparent vertical velocity arises as the ion rotates $\omega \tau$ before emitting a photon, with $\omega$ the ion gyro-frequency and $\tau$ the lifetime of the excited state of the transition. The gyro-orbit finite-lifetime effect scales with ion temperature and magnetic field strength. At the plasma edge the ion temperature is low, hence, the correction due to the gyro-orbit effect becomes less important. A correction for the gyro-orbit motion during the finite lifetime of the excited state has been calculated for the geometry of the new poloidal system using the analytic expressions derived by Solomon et $\mathrm{al}^{18}$ and performing a LOS-integration ${ }^{38}$. For a typical magnetic field of $2 \mathrm{~T}$ at the lowfield side of AUG, the gyro-frequency of $\mathrm{B}^{4+}$ is of the order $7 \cdot 10^{7} \mathrm{~Hz}$. The typical lifetime for the $\mathrm{B}^{4+}(n=7 \rightarrow 6)$ transition is on the timescale of ns, similar to the lifetime of 


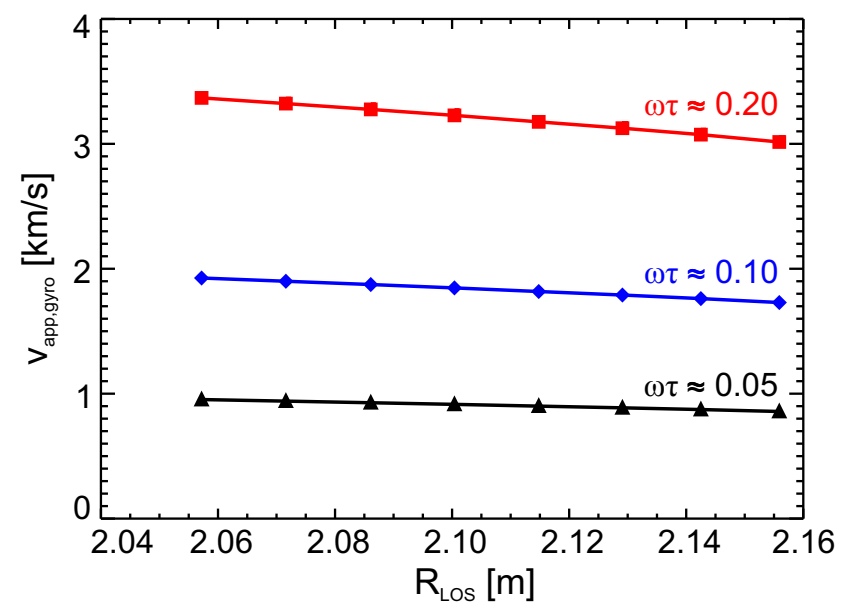

FIG. 13. Apparent velocity, $v_{a p p, g y r o}$, for $\mathrm{B}^{5+}$ as a function of the major radius of the LOS, $R_{L O S}$, of the poloidal system arising due to the gyro-motion effect for different values of $\omega \tau$ and an observed velocity of $v_{o b s}=20 \mathrm{~km} / \mathrm{s}$.

$\mathrm{C}^{5+}(n=8 \rightarrow 7)$. The Einstein coefficients for $\mathrm{B}^{4+}$ and $\mathrm{C}^{5+}$ have been calculated using the Cowan $\operatorname{code}^{39}$ (as provided by the Atomic Data Analysis Structure ADAS ${ }^{40}$ ), which are in agreement with those derived from Wiese et al. ${ }^{41}$ (deviations are smaller than $0.04 \cdot 10^{8}$ $\mathrm{s}^{-1}$ ). Calculating the vacuum radiative lifetime of both $\mathrm{B}^{4+}$ and $\mathrm{C}^{5+}$ yields similar values, i.e. $0.638 \mathrm{~ns}$ for $\mathrm{B}^{4+}$ and $0.552 \mathrm{~ns}$ for $\mathrm{C}^{5+}$, in good agreement with the vacuum radiative lifetime used in ref. ${ }^{37}$. Using a collisional-radiative model and allowing radiative decay from higher $n$ levels $^{37}$ an effective lifetime for $\mathrm{C}^{5+}$ was estimated as $0.7 \mathrm{~ns}$. Measurements on TFTR revealed an effective lifetime of $1.1 \mathrm{~ns}$ for the $\mathrm{C}^{5+} \operatorname{transition}^{37}$, while on DIII-D the experimentally derived lifetime was $1.7 \mathrm{~ns}^{18}$. For parameters at AUG and considering the $\mathrm{B}^{4+}$ transition these lifetimes translate to $\omega \tau \approx 0.05, \omega \tau \approx 0.08$ and $\omega \tau \approx 0.12$, respectively. A sensitivity study of increasing lifetimes has been performed and the results are shown in figure 13. The combined effect due to both the gyro-orbit motion and the CX cross-sections (section VB) is shown in figure 14, which illustrates the apparent velocity for $\mathrm{B}^{5+}$ as a function of the major radius of the LOS. For increasing values of $\omega \tau$ the apparent velocity appears to be higher for lower ion temperatures due to competing corrections arising from the CX cross-section and gyro-motion effect. For $\omega \tau=0$ the remaining effect is due to the energy-dependent CX cross-sections as discussed in section VB (see figure 11).

The combined CX cross-section and gyro-motion effect has been simulated for real impurity ion temperature and poloidal rotation profiles, as shown in figure 8. For this case only the 


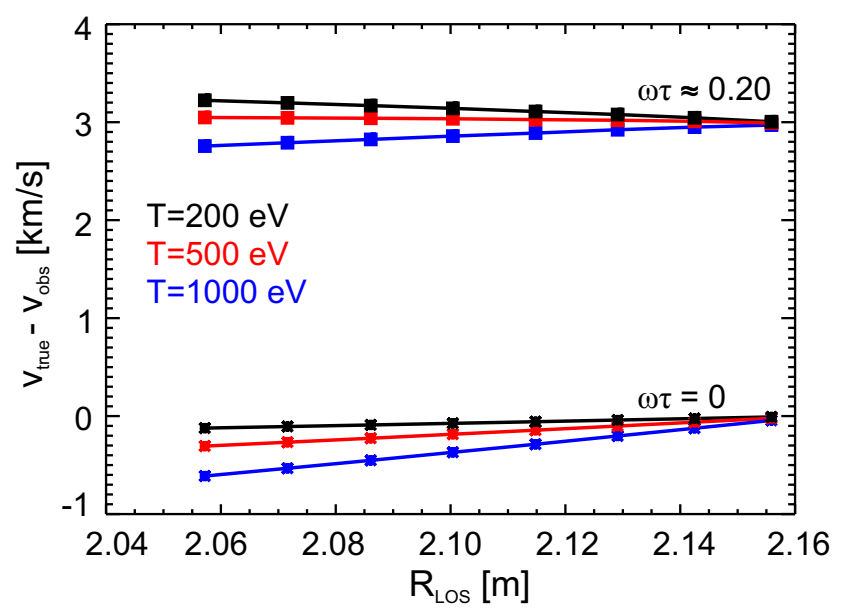

FIG. 14. Combined CX cross-section and gyro-motion effect for poloidal edge CXRS system: corrections for $\mathrm{B}^{5+}$ as a function of the major radius of the LOS, $R_{L O S}$, for two different values of $\omega \tau$ and for different ion temperatures. The curves are shown for an observed velocity of $v_{o b s}=20$ $\mathrm{km} / \mathrm{s}$. Note that for the case $\omega \tau=0$ (i.e. no gyro-motion effect) the impact of the energy dependence of the CX cross-sections is shown separately (as in figure 11).

first beam energy component has been considered. The effect of varying the lifetime of the $\mathrm{B}^{4+}$ transition is shown in figure 15 . Note that the theoretically and experimentally derived lifetimes $^{18,37}$ discussed above have been used for the simulations. In H-mode the poloidal

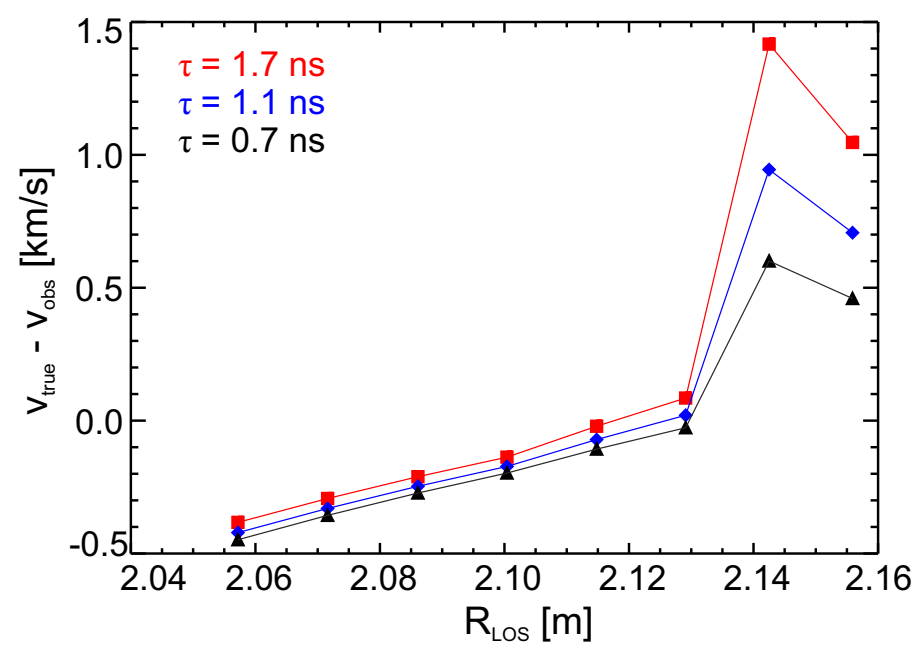

FIG. 15. Calculations for a real AUG case using measured impurity ion temperature and poloidal rotation profiles (as shown in figure 8): corrections for $\mathrm{B}^{5+}$ as a function of the major radius of the LOS, $R_{L O S}$, for different values of the lifetime $\tau$. 
rotation increases by an order of magnitude inside the ETB and competing corrections due to the CX cross-section and gyro-motion effect result in the profile shown in figure 15 .

The calculations presented in this section show that for the observed temperature and rotation ranges the corrections due to CX cross-section and gyro-motion effects on the measured spectra are small. The magnitude of the correction depends on the value of $\omega \tau$, which itself is uncertain within a factor of 2 . Therefore, the corrections are not included in the analysis. Due to the atomic physics effects an additional uncertainty of $\pm 0.5 \mathrm{~km} / \mathrm{s}$ arises for the poloidal rotation measurements, yielding a total uncertainty of $\pm 1.5 \mathrm{~km} / \mathrm{s}$.

\section{Correction due to Zeeman splitting}

Due to the presence of a magnetic field in tokamak plasmas the Zeeman effect needs to be taken into account. Each Zeeman component is a Gaussian itself with a Doppler width which represents the true ion temperature. When all of the components are added up a line-shape which is nearly Gaussian is produced. However, the width of this Gaussian overestimates the ion temperature ${ }^{1,42}$. Several methods are employed to account for the Zeeman splitting in CX spectra. Note that the total Zeeman pattern includes both the Zeeman and the fine structure contributions, i.e. the fine structure is also taken into account in the fitting process. At AUG, correction factors calculated as a function of the apparent ion temperature ${ }^{43}$ are used to account for the Zeeman broadening, assuming that the total line shape of the measured spectra is Gaussian. This assumption is valid at high temperatures and low magnetic fields,

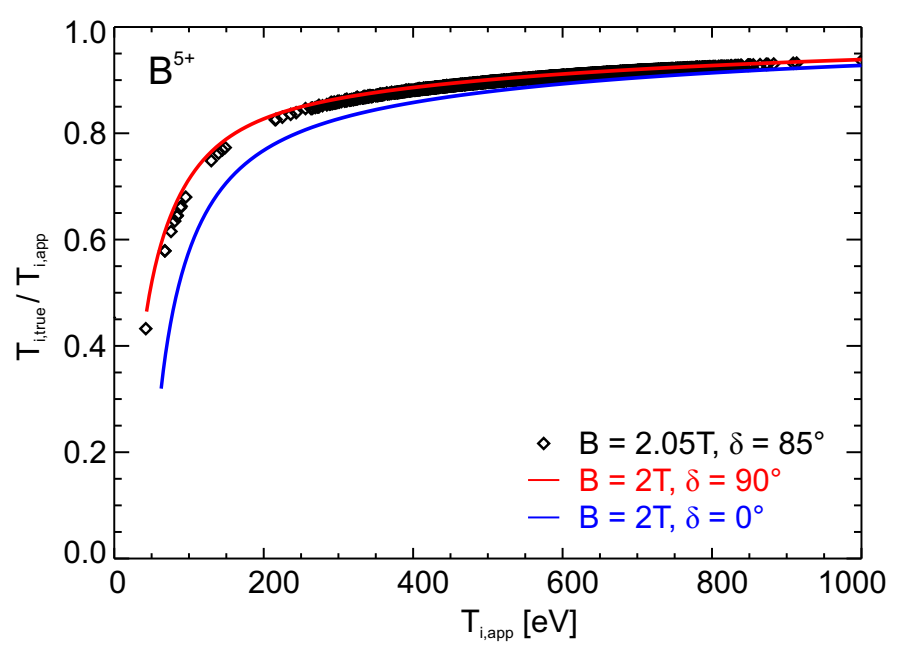

FIG. 16. Correction factor $T_{i, t r u e} / T_{i, a p p}$ for $\mathrm{B}^{5+}$ to account for Zeeman splitting in CX spectra. 
i.e. where the Doppler broadening is dominant compared to the Zeeman splitting. The Zeeman components are calculated assuming fully mixed populations of the $l$-states $(l$ being the orbital angular momentum quantum number). This assumption is valid in the ETB of AUG using an empirical formula ${ }^{44}$ for determining the critical plasma density at which a given transition is fully mixed. For the $\mathrm{B}^{5+}$ transition, a critical density of $1.3 \cdot 10^{19} \mathrm{~m}^{-3}$ is obtained which is lower than typical densities at the plasma edge of AUG indicating that the $l$-levels are fully mixed. Each component of the Zeeman pattern is convolved with a Gaussian which corresponds to the Doppler broadening at the real ion temperature. The resulting feature is well described by a single Gaussian with an apparent ion temperature $T_{i, a p p}$. Figure 16 shows the dependence of the correction factor for $\mathrm{B}^{5+}$ on the apparent ion temperature calculated for the LOS of the poloidal optical head (almost perpendicular view to the magnetic field) and a magnetic field of about $2 \mathrm{~T}$. The solid lines in blue and red show the theoretical curves calculated for $B=2 \mathrm{~T}$ and an angle of $0^{\circ}$ and $90^{\circ}$, respectively. The true ion temperature $T_{i, t r u e}$ is obtained by multiplying the measured apparent temperature with the correction factor. This procedure is performed for all of the CXRS diagnostics.

\section{SUMMARY}

The CXRS diagnostics at AUG have been upgraded and extended to provide impurity ion temperature, density and velocity profiles with increased temporal and radial resolution. The main core toroidal system and the edge toroidal system have been equipped with a new CCD camera and a new spectrometer enabling the measurement of 25 channels simultaneously. Additional lines of sight have been installed for the core toroidal system to provide detailed radial profiles $(\approx 2 \mathrm{~cm}$ resolution) with high temporal resolution down to $3.5 \mathrm{~ms}$. The edge toroidal system has a radial resolution of down to $3 \mathrm{~mm}$ and a temporal resolution of $2.2 \mathrm{~ms}$. Combining the measurements at the plasma core and at the edge allows full radial profiles to be obtained. The simultaneous measurement of impurity ion temperature, density and rotation velocity allows the cross-calibration of the different CXRS diagnostics. Good agreement is found between all systems. In addition, a new poloidal CXRS system has been installed which enables spatially (down to $5 \mathrm{~mm}$ ) and temporally $(1.9 \mathrm{~ms})$ resolved measurements of poloidal impurity ion rotation. Care has been taken to determine the wavelength calibrations with quite high accuracy. Deviations from the parabolic image of 
High-resolution CXRS at ASDEX Upgrade

a straight entrance slit, which would lead to an apparent velocity, have been quantified by measurements on spectral lamps. To minimize systematic uncertainties of the edge rotation measurements an additional wavelength correction is performed on a shot-to-shot basis. This method allows the determination of the wavelength calibration with uncertainties smaller than $1 \mathrm{~km} / \mathrm{s}$. Due to the atomic physics effects in the CX spectra additional uncertainties of $\pm 0.5 \mathrm{~km} / \mathrm{s}$ arise for the poloidal rotation measurements. Complementing the toroidal edge system with a poloidal view provides full edge rotation profiles at AUG, i.e. parallel and perpendicular to the magnetic field. To obtain detailed radial profiles of the edge transport barrier the radial plasma sweep technique is applied, thus enabling the alignment of the measurements of both edge CXRS diagnostics. Moreover, the measurements of the new poloidal edge system now enable the determination of the radial electric field from CXRS measurements at AUG needed for investigating the physics of the $\mathrm{L}-\mathrm{H}$ transition.

\section{ACKNOWLEDGMENTS}

This work was supported by the State Government of Bavaria through the 'Bayerisches Konjunkturpaket II'. The hardware upgrades for the toroidal core system I were funded in part by the EFDA 2009 Workprogram WP09-TGS-02/02.

\section{REFERENCES}

${ }^{1}$ R. J. Fonck, D. S. Darrow and K. P. Jaehnig. Determination of plasma-ion velocity distribution via charge-exchange recombination spectroscopy. Phys. Rev. A, 29(6):3288, 1984.

${ }^{2}$ M. Shimada et al. M. Shimada, D.J. Campbell, V. Mukhovatov, M. Fujiwara, N. Kirneva, K. Lackner, M. Nagami, V.D. Pustovitov, N. Uckan, J. Wesley, N. Asakura, A.E. Costley, A.J.H. Donné, E.J. Doyle, A. Fasoli, C. Gormezano, Y. Gribov, O. Gruber, T.C. Hender, W. Houlberg, S. Ide, Y. Kamada, A. Leonard, B. Lipschultz, A. Loarte, K. Miyamoto, V. Mukhovatov, T.H. Osborne, A. Polevoi and A.C.C. Sips. Chapter 1: Overview and summary. Nuclear Fusion, 47:S1, 2007.

${ }^{3}$ A. Herrmann, O. Gruber. Special Issue on ASDEX Upgrade. Fusion Science and Technology, 44(3), 2003. 
${ }^{4}$ T. Pütterich, E. Wolfrum, R. Dux, C. F. Maggi and ASDEX Upgrade Team. Evidence for Strong Inversed Shear of Toroidal Rotation at the Edge-Transport Barrier in ASDEX Upgrade. Phys. Rev. Lett., 102:025001, 2009.

${ }^{5} \mathrm{~K}$. Ida. Experimental studies of the physical mechanism determining the radial electric field and its radial structure in a toroidal plasma. Plasma Phys. Control. Fusion, 40:1429, 1998.

${ }^{6}$ F. Wagner, G. Becker, K. Behringer, D. Campbell, A. Eberhagen, W. Engelhardt, G. Fussmann, O. Gehre, J. Gernhardt, G. v. Gierke, G. Haas, M. Huang, F. Karger, M. Keilhacker, O. Klüber, M. Kornherr, K. Lackner, G. Lisitano, G. G. Lister, H. M. Mayer, D. Meisel, E. R. MüLLER, H. Murmann, H. Niedermeyer, W. Poschenrieder, H. Rapp, H. Röhr, F. Schneider, G. Siller, E. Speth, A. äbler, K. H. Steuer, G. Venus, O. Vollmer, Z. Yü. Regime of Improved Confinement and High Beta in Neutral-Beam-Heated Divertor Discharges of the ASDEX Tokamak. Phys. Rev. Lett., 49(19):1408, 1982.

${ }^{7}$ R. Dux, B. Geiger, R. M. McDermott, T. Pütterich, E. Viezzer and ASDEX Upgrade team. Impurity density determination using charge exchange and beam emission spectroscopy at ASDEX Upgrade. Conf. Proceedings of the $38^{\text {th }}$ EPS Conference on Plasma Physics, ECA Vol. 35G, P1.056, Strasbourg, 2011.

${ }^{8}$ B. Geiger, M. Garcia-Muñoz, W. W. Heidbrink, R. M. McDermott, G. Tardini, R. Dux, R. Fischer, V. Igochine and the ASDEX Upgrade Team. Fast-ion D-alpha measurements at ASDEX Upgrade. Plasma Phys. Control. Fusion, 53:065010, 2011.

${ }^{9}$ Princeton Instruments http://www.princetoninstruments.com.

${ }^{10}$ R. M. McDermott, C. Angioni, R. Dux, E. Fable, T. Pütterich, F. Ryter, A. Salmi, T. Tala, G. Tardini, E. Viezzer and the ASDEX Upgrade Team. Core momentum and particle transport studies in the ASDEX Upgrade tokamak. Plasma Phys. Control. Fusion, $53: 124013,2011$.

${ }^{11}$ C. Angioni, R. M. McDermott, E. Fable, R. Fischer, T. Pütterich, F. Ryter, G. Tardini and the ASDEX Upgrade Team. Gyrokinetic modelling of electron and boron density profiles of H-mode plasmas in ASDEX Upgrade. Nucl. Fusion, 51:023006, 2011.

${ }^{12}$ C. Angioni, R. M. McDermott, F. J. Casson, E. Fable, A. Bottino, R. Dux, R. Fischer, Y. Podoba, T. Pütterich, F. Ryter, E. Viezzer and ASDEX Upgrade Team. Intrinsic Toroidal Rotation, Density Peaking, and Turbulence Regimes in the Core of Tokamak Plasmas. Phys. Rev. Lett., 107:215003, 2011. 
${ }^{13}$ NIST Atomic Spectra Database. http://physics.nist.gov/PhysRefData/ASD/lines_form.html ${ }^{14}$ E. Viezzer, T. Pütterich, R. Dux, A. Kallenbach and the ASDEX Upgrade Team. Investigation of passive edge emission in charge exchange spectra at the ASDEX Upgrade tokamak. Plasma Phys. Control. Fusion, 53:035002, 2011.

${ }^{15}$ H. Zohm. Edge localized modes (ELMs). Plasma Phys. Control. Fusion, 38:105, 1996.

${ }^{16}$ J. Kim, K. H. Burrell, P. Gohil, R. J. Groebner, Y.-B. Kim, H. E. St. John, R. P. Seraydarian and M. R. Wade. Rotation characteristics of main ions and impurity ions in H-mode tokamak plasma. Phys. Rev. Lett., 72(14):2199, 1994.

${ }^{17}$ K. H. Burrell, D. H. Kaplan, P. Gohil, D. G. Nilson, R. J. Groebner, D. M. Thomas. Improved charge coupled device detectors for the edge charge exchange spectroscopy system on the DIII-D tokamak. Rev. Sci. Instrum., 72(1):1028, 2001.

${ }^{18}$ W. M. Solomon, K. H. Burrell, P. Gohil, R. J. Groebner, L. R. Baylor. Extraction of poloidal velocity from charge exchange recombination spectroscopy measurements. Rev. Sci. Instrum., 75(10):3481, 2004.

${ }^{19}$ R. M. McDermott, B. Lipschultz, J. W. Hughes, P. J. Catto, A. E. Hubbard, I. H. Hutchinson, R. S. Granetz, M. Greenwald, B. LaBombard, K. Marr, M. L. Reinke, J. E. Rice, D. Whyte, and Alcator C-Mod Team. Edge radial electric field structure and its connections to H-mode confinement in Alcator C-Mod plasmas. Phys. Plasmas, 16:056103, 2009.

${ }^{20}$ N. Hawkes, D. V. Bartlett, D. J. Campbell, N. Deliyanakis, R. M. Giannella, P. J. Lomas, N. J. Peacock, L. Porte, A. Rookes and P. R. Thomas. Evolution of edge radial electric field at the L to H transition in JET. Plasma Phys. Control. Fusion, 38(8):1261, 1996.

${ }^{21}$ K. Crombé, Y. Andrew, I. Coffey, P. De Vries, C. Giroud, N. C. Hawkes, E. Joffrin, D. C. McDonald, A. Murari, J. Ongena, F. Sattin, M. Valisa, K. D. Zastrow and EFDA-JET contributors. Improved measurements of impurity ion poloidal rotation velocity in JET. Conf. Proceedings of the $30^{\text {th }}$ EPS Conference on Contr. Fusion and Plasma Physics, ECA Vol. 27A, P-1.55, St. Petersburg, 2003.

${ }^{22}$ Y. Andrew, N. C. Hawkes, K. Crombé and JET EFDA contributors. Improved charge exchange spectroscopy on the Joint European Torus for ion temperature and rotation velocity profiles. Rev. Sci. Instrum., 77:10E913, 2006.

${ }^{23}$ R. E. Bell, F. M. Levinton, S. H. Batha, E. J. Synakowski and M. C. Zarnstorff. Poloidal Rotation in TFTR Reversed Shear Plasmas. Phys. Rev. Lett., 81(7):1429, 1998. 
${ }^{24} \mathrm{C}$. Busch. Volume 50 of 'Schriften des Forschungszentrums Juelich', Energy Technology edition, ISBN 3-89336-433-1, Forschungszentrum Juelich GmbH, 2006.

${ }^{25}$ A. R. Field, J. McCone, N. J. Conway, M. Dunstan, S. Newton and M. Wisse. Comparison of measured poloidal rotation in MAST spherical tokamak plasmas with neo-classical predictions. Plasma Phys. Control. Fusion, 51:105002, 2009.

${ }^{26}$ R. E. Bell, R. Andre, S. M. Kaye, R. A. Kolesnikov, B. P. LeBlanc, G. Rewoldt, W. X. Wang and S. A. Sabbagh. Comparison of poloidal velocity measurements to neoclassical theory on the National Spherical Torus Experiment. Phys. Plasmas, 17:082507, 2010.

${ }^{27}$ K. Ida, S. Kado and Y. Liang. Measurements of poloidal rotation velocity using charge exchange spectroscopy in a large helical device. Rev. Sci. Instrum., 71(6):2360, 2000.

${ }^{28}$ E. Wolfrum, R. Fischer, B. Langer, T. Pütterich, G. D. Conway, D. P. Coster, R. Dux, J.C. Fuchs, M. Gemisic Adamov, N. Hicks, L. D. Horton, C. Konz, B. Kurzan, K. Lackner, C. F. Maggi, P. J. McCarthy, M. Reich, J. Schweinzer, W. Suttrop, C. Tröster and ASDEX Upgrade Team. Pedestal Studies at ASDEX Upgrade. Conf. Proceedings of the $22^{\text {nd }}$ IAEA Fusion Energy Conference, EX/P3-7, Geneva, 2008.

${ }^{29}$ B. Kurzan, H. D. Murmann. Edge and core Thomson scattering systems and their calibration on the ASDEX Upgrade tokamak. Rev. Sci. Instrum., 82:103501, 2011.

${ }^{30} \mathrm{~W}$. Suttrop. Practical Limitations to Plasma Edge Electron Temperature Measurements by Radiometry of Electron Cyclotron Emission. IPP Report 1/306, Max-Planck-Institute for Plasma Physics, Garching, Germany, 1997.

${ }^{31}$ J. Neuhauser, D. Coster, H. U. Fahrbach, J. C. Fuchs, G. Haas, A. Herrmann, L. Horton, M. Jakobi, A. Kallenbach, M. Laux, J. W. Kim, B. Kurzan, H. W. Müller, H. Murmann, R. Neu, V. Rohde, W. Sandmann, W. Suttrop, E. Wolfrum and the ASDEX Upgrade Team. Transport into and across the scrape-off layer in the ASDEX Upgrade divertor tokamak. Plasma Phys. Control. Fusion, 44:855, 2002.

${ }^{32}$ R. Fischer, E. Wolfrum, J. Schweinzer and the ASDEX Upgrade Team. Probabilistic lithium beam data analysis. Plasma Phys. Control. Fusion, 50:085009, 2008.

${ }^{33}$ M. Willensdorfer, E. Wolfrum, R. Fischer, J. Schweinzer, M. Sertoli, B. Sieglin, G. Veres, F. Aumayr and the ASDEX Upgrade Team. Improved chopping of a lithium beam for plasma edge diagnostic at ASDEX Upgrade. Rev. Sci. Instrum., 83:023501, 2012.

${ }^{34}$ ITER Physics Expert Group on Confinement and Transport, ITER Physics Expert Group on Confinement Modelling and Database and ITER Physics Basis Editors. Chapter 2: 
High-resolution CXRS at ASDEX Upgrade

Plasma confinement and transport. Nucl. Fusion, 39:2175, 1999.

${ }^{35}$ R. E. Bell. Exploiting a transmission grating spectrometer. Rev. Sci. Instrum., 75(10):4158, 2004.

${ }^{36}$ M. von Hellermann, P. Breger, J. Frieling, R. K“nig, W. Mandl, A. Maas and H. P. Summers. Analytical approximation of cross-section effects on charge exchange spectra observed in hot fusion plasmas. Plasma Phys. Control. Fusion, 37:71, 1995.

${ }^{37}$ R. E. Bell and E. J. Synakowski. New understanding of poloidal rotation measurements in a tokamak plasma. AIP Conf. Proc. 547, 547:39, 2000.

${ }^{38}$ K. Crombé. Spectroscopic studies of Impurity Ion Dynamics on the JET and TEXTOR Tokamaks. PhD thesis, ISBN 90-8578-033-0, Ghent University, available at http://hdl.handle.net/1854/5551, 2006.

${ }^{39}$ R. D. Cowan. The Theory of Atomic Structure and Spectra. University of California Press, ISBN 9780520038219, 1981.

${ }^{40}$ H. P. Summers. ADAS User Manual 2.6, available at http://www.adas.ac.uk/manual.php, 2004 .

${ }^{41}$ W. L. Wiese, M. W. Smith and B. M. Glennon. Atomic Transition Probabilities, Volume I Hydrogen Through Neon, A Critical Data Compilation, NSRDS-NBS 4. National Standard Reference Data Series, National Bureau of Standards 4, U. S. Government Printing Office, Washington, D. C., 1966.

${ }^{42} \mathrm{~A}$. Blom and C. Jupén. Parametrization of the Zeeman effect for hydrogen-like spectra in high-temperature plasmas. Plasma Phys. Control. Fusion, 44:1229, 2002.

${ }^{43}$ J. D. Hey, Y. T. Lie, D. Rusbüldt, E. Hintz. Spectroscopic Measurements of Ion Temperatures in the Boundary Layer of TEXTOR and their Interpretation. Conf. Proceedings of the $20^{t h}$ EPS Conference on Contr. Fusion and Plasma Physics, ECA Vol. 17C/III, p. 1111, Lisbon, 1993.

${ }^{44}$ D. H. Sampson. On statistical equilibrium among the sublevels of hydrogenic atoms and ions. J. Phys. B: At. Mol. Phys., 10:749, 1977. 